\title{
On Small Disturbances of Plane Couette Flow
}

\author{
Wolfgang Wasow ${ }^{2}$
}

\begin{abstract}
The Orr-Sommerfeld equation for the function $\phi(y)$, which appears in the component $\phi(y) \exp \{i \alpha(x-c t)\}$ of the stream function, is analyzed in detail for the case of plane Couette flow. A set of solutions is found whose asymptotic behavior as $\alpha R \rightarrow \infty$ can be calculated in the whole complex $y$-plane ( $R$ is the Reynolds number). A disturbance with given $\alpha$ is proved stable, for any $\alpha$, if $\alpha R$ is sufficiently large. In addition, an investigation is made of the asymptotic properties of an allied equation for which, in contradistinction to the singular Couette case, an inner friction layer does exist.
\end{abstract}

\section{Introduction}

The purpose of this paper is to analyze mathematically some questions connected with the stability of plane Couette flow.

If the space between two infinite parallel planes at constant distance in time is filled with a viscous, incompressible fluid, and if one plane is at rest while the other moves with constant velocity, then the simplest flow compatible with the Navier-Stokes equations and these boundary conditions is the stationary parallel motion with linear velocity profile. This is plane Couette flow. Experiments indicate, $\left[1\right.$, p. 185], ${ }^{3}$ that this flow, just as most other viscous flows, becomes unstable, that is, turbulent, for high Reynolds numbers.

The oldest and best developed theory of hydrodynamic stability for plane parallel flows, not only for Couette flow, studies the stability of disturbances so small that they can be regarded as solutions of a linearized differential equation. It is further assumed that the stream functions of such disturbances can be obtained by superposition of functions of the form

$$
\phi(y) e^{i \alpha(x-c t)},
$$

if the main flow is taken to be parallel to the $x$-axis. Here, $\alpha$ is positive, $c$ and $\phi(y)$ are complex, and $t$ is the time. The references $[1,2]$ contain fuller accounts of this theory. It reduces the stability problem to eigenvalue problems for the differential equation

$\phi^{(4)}-2 \alpha^{2} \phi^{\prime \prime}+\alpha^{4} \phi-i \alpha R\left[(w-c)\left(\phi^{\prime \prime}-\alpha^{2} \phi\right)-w^{\prime \prime} \phi\right]=0$,

where $w=w(y)$ is the velocity of the main flow and $R$ denotes a Reynolds number of the flow.

A solution (eigenfunction) of (2) that satisfies the homogeneous boundary conditions of a particular flow problem under consideration yields a stable or unstable disturbance of that flow according as the corresponding eigenvalue $c$ has a negative or positive imaginary part. For a given value of $R$ the flow is

1 The preparation of this paper was sponsored (in part) by the Office of Naval Research, USN.

2 National Bureau of Standards, Los Angeles, Calif.

3 Figures in brackets indicate the literature references at the end of this paper. stable, if one has $\operatorname{Im} c \leq 0$ for all possible eigenvalues. Otherwise the flow is unstable.

This approach has been very successful for a number of velocity profiles. It has led to critical Reynolds numbers that are in good agreement with experimental evidence, $[4,5,6]$. A mathematically difficult part of this method is the integration of the differential equation (2). For large values of $\alpha R$ asymptotic methods can be used whose validity has been established in [7, 8 and 10].

Couette flow, however, although it has the simplest velocity profile, plays an exceptional role in these theories. First, the proofs of [7,8 and 10] do not cover this case. Second, all investigations seem to indicate that Couette flow is stable, in the sense specified above, at all Reynolds numbers, although no mathematically complete proof of this has been given (cf. [2], p. 256). Third, in contrast to other profiles, no so-called "inner friction layers" [3, 8] occur in the theory of Couette flow.

This paper will consist of three parts, corresponding to these three points.

(a) The first part (section 2) is in the nature of a supplement to [9] and [10]. A fundamental system of the differential equation (2) for the case of Couette flow is constructed whose asymptotic behavior, as $\alpha R \rightarrow \infty$, can be calculated in the whole complex $y$-plane.

(b) In the second part (section 3) the eigenvalues $c$ of (2), corresponding to possible disturbances of the flow, are studied as functions of $\alpha$ and $\alpha R$. It is proved that for any given $\alpha$ a disturbance of type (1) of Couette flow is stable, if $\alpha R$ is sufficiently large. If the physically plausible conjecture is accepted, that above a certain frequency $\alpha$ there is always stability (cf. [3], part III, p. 284), the stronger statement can be made that, uniformly in $\alpha$, there is a lower bound for $\alpha R$ above which the flow is stable.

On the other hand there are the inequalities of Synge ([2], p. 258), which imply the stability of Couette flow for sufficiently small values of $R$. Even if taken together, these results do not, of course, constitute a complete mathematical proof of stability. But the author believes that they go farther towards this goal than previous efforts.

C. Morawetz has shown [13] that the possible eigenvalues $c$ of the stability problem fall asymptotically into three classes: (1) Eigenvalues that 
approach those of the limiting inviscid problem as $\alpha R \rightarrow \infty$. The corresponding eigenfunctions approximate those of the inviscid problem except possibly in certain "friction layers." (2) An infinity of eigenvalues lying increasingly close to a certain curve in the $c$-plane as $\alpha R \rightarrow \infty$. They are in no way related to the inviscid differential equation. The corresponding disturbances are always stable. (3) Eigenvalues that approach the points $w\left(y_{1}\right)$ or $w\left(y_{2}\right)$, as $\alpha R \rightarrow \infty$, where $y_{1}$ and $y_{2}$ are the ordinates of the flow boundaries. The actual existence or stability of eigenvalues of this third kind was not discussed in [13].

Since no eigenvalues of the first kind exist for plane Couette flow, its asymptotic stability, in the sense stated above, is proved if the disturbances of the third kind are shown to be stable for sufficiently large values of $R$. This will be done in section 3.

(c) In all cases examined so far, the actually possible disturbances of laminar flows are approximations to solutions of the inviscid differential equation, except, in general, in certain sectors of the complex $y$-plane, where they diverge exponentially, as $\alpha R \rightarrow \infty$ (cf. $[8,9,10])$. The results of section 2 show that no such sectors of divergence exist in the Couette case.

In section 4 , the mathematical reason for this absence is analyzed. Although not immediately applicable to physical problems, the results of that section may turn out to be of more than purely mathematical interest.

\section{Solution of the Differential Equation}

In terms of the dimensionless quantities used in (2), the velocity profile of Couette flow is, for an appropriate definition of $R$,

$$
w(y)=y \text {. }
$$

The further substitutions

$$
\begin{gathered}
z=y-c, \\
\lambda=(-\alpha R i)^{\frac{1}{2}}, \\
\phi(y)=u(z)
\end{gathered}
$$

change (2) into the simpler form

$$
u^{(4)}-2 \alpha^{2} u^{\prime \prime}+\alpha^{4} u+\lambda^{2} \approx\left(u^{\prime \prime}-\alpha^{2} u\right)=0 .
$$

The theory of [10] does not apply to this differential equation, because condition (3.3) of that paper is not satisfied. But (7) can be treated directly by the method of Laplace transformation used in [9].

By a straightforward application of Laplace's method, it is seen that contour integrals of the form

$$
u(z, \lambda)=\int_{C}\left(t^{2}-\alpha^{2}\right)^{-1} e^{\lambda^{-2}\left(t^{3} / 3-\alpha_{z}^{2} t\right)+z t} d t
$$

are solutions of (7), provided $C$ is a path that is either closed or that extends to infinity in such a

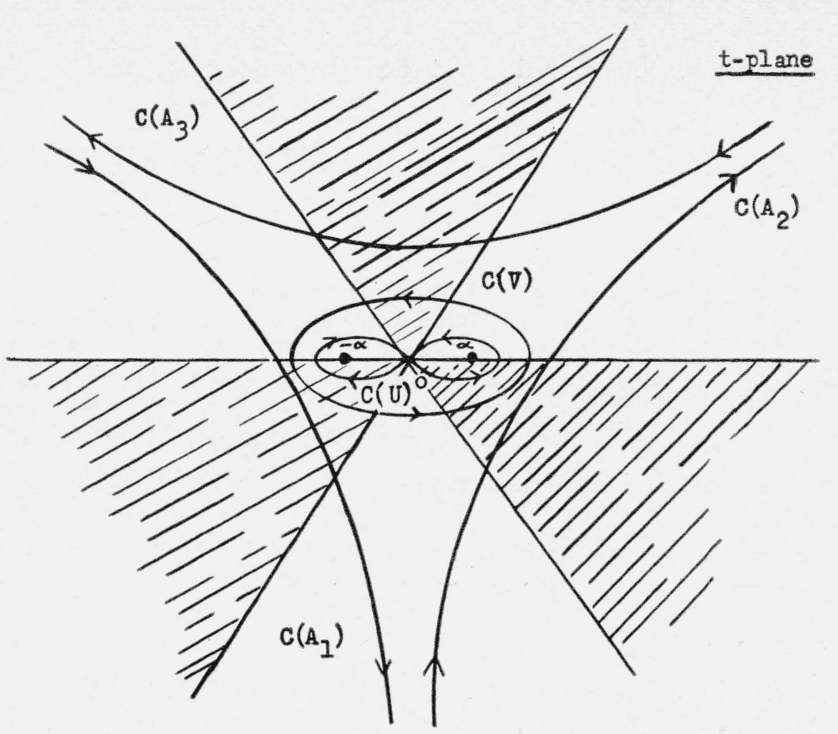

Figure 1.

way that $\operatorname{Re}\left(\lambda^{-2} t^{3}\right)<0$. Five such paths are indicated in the figure 1 , where the sectors in which $\operatorname{Re}\left(\lambda^{-2} t^{3}\right)>0$ have been shaded. The paths $C\left(A_{j}\right)$ $(j=1,2,3)$ are the same as in [9 and 10]. The solutions corresponding to these contours will be called $A_{j}(z, \lambda), U(z, \lambda)$ and $V(z, \lambda)$. Clearly,

$$
A_{1}+A_{2}+A_{3}=V \text {. }
$$

Let $S$ denote any bounded neighborhood of the origin in the z-plane. Then a short calculation shows that, in $S$,

$$
\begin{aligned}
& U(z, \lambda)=\frac{2 \pi i}{\alpha} \cosh \alpha z \\
& V(z, \lambda)=\frac{2 \pi i}{\alpha} \sinh , \alpha z
\end{aligned}
$$

The asymptotic calculation of the solutions $A_{j}(z, \lambda)$ proceeds exactly as in [9]. It is amply sufficient, therefore, to restate those results.

Let $\xi$ and $\eta$ be defined by

$$
\xi=\frac{2}{3} \lambda(-z)^{3 / 2}, \quad \eta=\lambda^{2 / 3} z
$$

and denote by $C_{k}(k=1,2,3)$ the three rays $\operatorname{Re} \xi=0$ numbered so that

$$
\arg z=\frac{4 k}{3} \pi-\frac{2}{3} \arg \lambda, \quad \text { on } C_{k} \text {. }
$$

The closed sector of $S$ bounded by $C_{j},(j \neq k)$, and of central angle $2 \pi / 3$ may be called $S_{k}$. If $\xi_{0}$ is any arbitrary positive constant, one has then

(a) For $|\xi| \geq \xi_{0}$, $\approx$ in $S-C_{k}$,

$$
A_{k}(z, \lambda)=\sqrt{\pi} \lambda^{-2 / 3}\left(\frac{3}{2} \xi\right)^{-5 / 6} e^{\xi}\left[1+O\left(\xi^{-1}\right)\right]
$$


Here, the determination of $\xi$ for which $\operatorname{Re} \xi \leq 0$ in $S_{k}$ and $R e \xi>0$ in $S-S_{k}$ must be taken.

(b) For $|\xi| \leq \xi_{0}$,

$$
A_{k}(z, \lambda)=\lambda^{-2 / 3} \int_{C^{*}\left(A_{k}\right)} t^{-2} e^{t_{\eta}+t^{3} / 3} d t+O\left(\lambda^{-4 / 3}\right),
$$

where $C^{*}\left(A_{k}\right)$ is obtained from $C\left(A_{k}\right)$ by a rotation through $-2 / 3$ arg $\lambda$.

(c) Near $C_{k}$ the asymptotic behavior of $A_{k}$ for $|\xi| \geq \xi_{0}$ follows immediately from (9), (11) and (14).

These asymptotic relations may be indefinitely formally differentiated. Without much additional effort it can be shown that (14) and (15) are first approximations of infinite asymptotic series progressing in powers of $\xi^{-1}$ and $\lambda^{-2 / 3}$, respectively.

The angle of $\xi^{-5 / 6}$ in formula (14) is $\pi / 2-2 \pi k / 3$ $(\bmod 2 \pi)$ at the points of the angular bisector of $S-C_{k}$. At the other points of the domain $S-C_{k}$, it is then obtained by analytic continuation. The angle of $\lambda^{-2 / 3}$ is $-2 / 3 \arg \lambda$. The angle of $\lambda$ will be fixed in accordance with (5) by setting

$$
\arg \lambda=\frac{3 \pi}{4} .
$$

\section{Stability Problem}

The coordinate system and the definition of the Reynolds number can, and will, be chosen so that the boundaries of the flow are at

$$
y_{1}=0, \quad y_{2}=1 \text {. }
$$

The boundary conditions for the disturbance are then ([3], p. 134)

$$
\phi(0)=\phi(1)=\phi^{\prime}(0)=\phi^{\prime}(1)=0 .
$$

In terms of the variables introduced in (4), (5) and (6) this becomes

$$
u(-c)=u(1-c)=u^{\prime}(-c)=u^{\prime}(1-\mathrm{c})=0 .
$$

The differential equation (7) with the boundary condition (19) constitutes the eigenvalue problem of plane Couette flow. In view of the results of [13] described in section 1 only those eigenvalues $c=c(\alpha, \lambda)$ need to be studied, for which either

or

$$
\lim _{\lambda \rightarrow \infty} c(\alpha, \lambda)=0
$$

$$
\lim _{\lambda \rightarrow \infty} c(\alpha, \lambda)=1 \text {. }
$$

(The notation differs from that of [13] in that there $\lambda=\sqrt{\alpha R}$, and that the names of the sectors $S_{1}$ and $S_{2}$ are interchanged.) The case (20) will be considered first. Using (13) and (16), the sectors $S_{k}$ are seen to have the appearance indicated by the figure 2 .

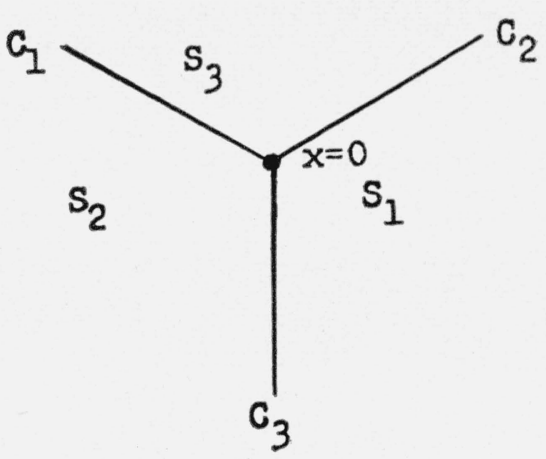

Figure 2.

A convenient fundamental system for the present discussion is formed by the functions $A_{1}, A_{2}, U, V$. Then the eigenvalues are the solutions of the equation

$F(\alpha, c, \lambda) \equiv\left|\begin{array}{llll}A_{1}(-c) & A_{2}(-c) & U(-c) & V(-c) \\ A_{1}^{\prime}(-c) & A_{2}^{\prime}(-c) & U^{\prime}(-c) & V^{\prime}(-c) \\ A_{1}(1-c) & A_{2}(1-c) & U(1-c) & V(1-c) \\ A_{1}^{\prime}(1-c) & A_{2}^{\prime}(1-c) & U^{\prime}(1-c) & V^{\prime}(1-c)\end{array}\right|=0$.

Two ranges of $c$ have to be distinguished according as $\eta_{c}=\lambda^{2 / 3} c$ remains bounded or is unbounded as $\lambda \rightarrow \infty$. If $\eta_{c}$ is very large, the asymptotic expressions (14) can be used at both end points, and arguments analogous to those employed in [13] show that the corresponding value of $c$ cannot have positive imaginary part.

If $\eta_{c}$ remains bounded as $\lambda \rightarrow \infty$ the expressions (15) are to be used at $z=1-c$ and the expressions (14) for $z=-c$, that is, $\eta=\eta_{c}$. Setting, for abbreviation

$$
g_{k}(\eta)=\int_{C^{*}\left(A_{k}\right)} t^{-2} e^{t \eta+t^{3} / 3} d t
$$

one finds, after a short calculation, that $F(\alpha, c, \lambda)=0$ becomes, in this case,

$$
g_{1}^{\prime}\left(\eta_{c}\right)+\lambda^{-2 / 3} E\left(\alpha, \lambda, c, \eta_{c}\right)=0
$$

where $E\left(\alpha, \lambda, c, \eta_{c}\right)$ is bounded for fixed $\alpha$, as $\lambda \rightarrow \infty$, provided $\eta_{c}$ remains bounded. Using Rouché's theorem, as in [13], it can be proved that, if $\eta=\eta_{0}$ is a root of the equation

$$
g_{1}^{\prime}(\eta)=0,
$$

then

that is,

$$
\eta_{c}=\eta_{0}+O\left(\lambda^{-2 / 3}\right),
$$

$$
c(\alpha, \lambda)=-\lambda^{-2 / 3} \eta_{0}+O\left(\lambda^{-4 / 3}\right) .
$$


If $c(\alpha, \lambda) \rightarrow 1$, the calculations are analogous. The roles of the two end points are interchanged, and $A_{1}$ is dominant at the left end point. If $\eta_{1}$ is a root of the equation

$$
g_{2}(\eta)=0,
$$

then there exist solutions of $F=0$ of the form

$$
c(\alpha, \lambda)=1-\lambda^{-2 / 3} \eta_{1}+O\left(\lambda^{-4 / 3}\right) .
$$

The preceding results reduce the question of the sign of $\operatorname{Im} c$ for large Reynolds numbers to inequalities concerning the position of the roots of eq (25) and (28). In order to study this question, it is convenient to introduce the variable

$$
s=\omega \eta,
$$

where

$$
\omega=e^{2 \pi i / 3},
$$

and the function

$$
\psi(s)=\frac{1}{2 \pi i} g_{1}^{\prime}(\eta) .
$$

Since the definition (23) implies that

$$
g_{2}^{\prime}(\eta)=g_{1}^{\prime}(\omega \eta)=g_{3}^{\prime}\left(\omega^{2} \eta\right)
$$

all three functions $g_{k}^{\prime}(\eta)$ can be simply expressed in terms of $\psi(s)$.

The values $c(\alpha, \lambda)$ given by (27) have, for sufficiently large $\lambda$, positive imaginary parts, if, and only if, $\operatorname{Re} \eta_{0}>0$, that is if the zeros of $\psi(s)$ lie in the half-plane $\pi / 6<\arg s<7 \pi / 6$. Similarly, the values $c(\alpha, \lambda)$ given by $(29)$ have, for large $\lambda$ positive imaginary parts, if $\operatorname{Re} \eta_{1}>0$. From (33) it follows that the necessary and sufficient condition for this to happen is that the zeros of $\psi(s)$ lie in the half-plane $-7 \pi / 6<\arg s<-\pi / 6$. Hence all disturbances are stable, for sufficiently large Reynolds numbers, if all roots of $\psi(s)$ lie in the sector $\arg s<\pi / 6$.

In order to prove that this is indeed the case, a few properties of the function $\psi(s)$ must first be collected.

From (23), (30), (32) and (33; , it is seen that the integral representation

$$
\psi(s)=\frac{1}{2 \pi i} g_{3}^{\prime}(s)=\frac{1}{2 \pi i} \int_{C^{*}\left(A_{3}\right)} t^{-1} e^{t s+t^{3} / 3} d t
$$

is valid. By a calculation very similar to that of $A_{3}(x, \lambda)$ in [9] asymptotic expressions for $\psi(s)$ can be derived when $|s|$ is large. The calculation results in the following formulas

$$
\psi(s)=\left\{\begin{array}{l}
\frac{1}{2 \sqrt{\pi}}(-s)^{-3 / 4} e^{-\frac{2}{3}(-s)^{3 / 2}}\left[1+O\left(s^{-1}\right)\right] \\
1-\frac{1}{\sqrt{\pi}} s^{-3 / 4} \cos \left(\frac{2}{3} s^{3 / 2}+\frac{\pi}{4}\right) \\
+O\left(s^{-7 / 4} \exp \left\{\frac{2}{3}\left|(\operatorname{Re} s)^{3 / 2}\right|\right\}\right) .
\end{array}\right.
$$

Here the first formula is valid outside any sector containing the positive real axis, and the second one for $|\arg s|<2 \pi / 3$. In the common part of these two sectors the two expressions are equivalent. In (35) the powers $(-s)^{-3 / 4}$ and $2(-s)^{3 / 2} / 3$ are to be taken positive on the negative real axis and continued analytically into the whole sector of validity. In (36) the powers $s^{-3 / 4}$ and $s^{3 / 2}$ are positive on the positive real axis and must be continued analytically into $|\arg s|<2 \pi / 3$. The derivation of (35) and (36) is omitted except for the remark that actually only formula (35) need be proved directly, for from formula (33) one obtains analogous formulas for $g_{1}^{\prime}(\eta)$ and $g_{2}^{\prime}(\eta)$, and the identity

$$
g_{1}^{\prime}(\eta)+g_{2}^{\prime}(\eta)+g_{3}^{\prime}(\eta)=2 \pi i
$$

which follows directly from (23), leads then to (36).

Differentiation of the relation (34) shows that

$$
\psi^{\prime}(s)=A i(-s)
$$

where $A i(s)$ is Airy's integral (cf. [14] and [8]). By (35), the function $\psi(s)$ tends to zero as $s \rightarrow \infty$ in the sector $2 \pi / 3<\arg s<4 \pi / 3$. Integrating (38) one finds, therefore,

$$
\psi(s)=\int_{\Gamma_{s}} A i(-t) d t .
$$

Where $\Gamma_{s}$ is any path starting at infinity in the sector $2 \pi / 3<\arg t<4 \pi / 3$ and ending at $t=s$. Since the Airy integral is real for real $s$, it follows from (39) that $\psi(s)$ is also real on the real axis.

Equations (33) and (37) imply that $\psi(0)=1 / 3$. Hence, (39) may be replaced by

$$
\psi(s)=1 / 3+\int_{0}^{s} A i(-t) d t .
$$

Since Airy's integral is related to Hankel's function by the identity

$$
A_{i}(s)=\frac{\omega}{2 \sqrt{3}} \sqrt{s} H_{1 / 3}^{(1)}\left(\frac{2 i}{3} s^{3 / 2}\right),
$$

where the branch of the three-valued Hankel function is determined by the condition that $A i(s)$ must be real on the real axis, the integral in (39) can be transformed into

$$
\psi(s)=\frac{\omega}{2 i \sqrt{3}} \int_{L_{\zeta}} H_{1 / 3}^{(1)}(\tau) d \tau .
$$

Here

$$
\zeta=\frac{2}{3} i(-s)^{3 / 2}
$$

and $L_{\zeta}$ is a path that begins at $\tau=\zeta$ and goes to infinity in the upper half plane. As the function $H_{1 / 3}^{(1)}(\tau)$ has a branch point at $\tau=0$, while $\psi(s)$ is single valued, the position of $L_{\zeta}$ with respect to the origin must be specified. If

$$
0 \leq \arg s<\pi \text {, }
$$


and the determination of $\zeta$ in (42) is taken for which

$$
-\pi<\arg \zeta \leq \frac{\pi}{2}
$$

a short argument, omitted here, shows that $L_{\zeta}$ must be in $-\pi<\arg \tau<\pi / 2$.

As to the zeros of $\psi(s)$, it follows from the asymptotic representation (35) that outside a sufficiently large circle they must all lie arbitrarily close to the positive real axis.

On the other hand, $\psi(s)$ has no real zeros. To show this, one can use the fact that $\psi(s)$ is a solution of the differential equation

$$
\psi^{\prime \prime \prime}+s \psi^{\prime}=0 .
$$

This can be verified, either by means of (34), or by using (39) and the differential equation for Airy's function. If $(45)$ is multiplied by $2 \psi$, the relation

$$
\frac{d}{d s}\left(2 \psi^{\prime \prime} \psi-\psi^{\prime 2}\right)+s \frac{d}{d s}\left(\psi^{2}\right)=0
$$

is obtained. Assume, now, that $s=a$ is a zero of $\psi(s)$. Integrating (46) from $-\infty$ to $a$, it follows then that

$$
\psi^{\prime 2}(a)+\int_{-\infty}^{a} \psi^{2}(t) d t=0 .
$$

This is impossible for real $a$, because $\psi(s)$ is real on the real axis.

After these preparations, it will now be proved that the sector $\pi / 6 \leq \arg s \leq 11 / 6 \pi$ contains no zeros of $\psi(s)$. Since $\psi(s)$ assumes conjugate values at conjugate points, only the sector $\pi / 6 \leq \arg s \leq \pi$ needs to be investigated.

Consider first the portion of this sector for which

$$
|s| \geq r_{0}
$$

If $r_{0}$ is chosen so large that the remainder denoted by $O\left(s^{-1}\right)$ in formula (35) is numerically less than unity, for $|s| \geq r_{0}$, the absence of zeros of $\psi(s)$ in the domain under consideration is a direct consequence of Rouché's theorem. An appraisal of the remainder can be based on the following lemma, which is a special case of a more general result of Weber [16, 17, p. 212].

Lemma 1. If $R(s)$ is defined by

then

$$
H_{1 / 3}^{(1)}(s)=\left(\frac{2}{\pi s}\right)^{\frac{1}{2}} e^{i(s-5 \pi / 12)}[1+R(s)]
$$

$$
|R(s)| \leq \frac{5 \pi}{72}\left|1-\frac{11}{12|s|}\right|^{-17 / 3}\left(1+\frac{8}{3|s|}\right)^{2}|s|^{-1}
$$

for $-\pi<\arg s<2 \pi$.
Substitution of (48) into (41) followed by an integration by parts leads to the expression

$\psi(s)=\frac{e^{-i \pi / 4}}{\sqrt{6 \pi}}\left[\zeta^{-1 / 2} e^{i \zeta}-\int_{L_{\zeta}} \tau^{-3 / 2} e^{i \tau}\left(\frac{1}{2}+i R(\tau) \tau\right) d \tau\right]$.

From (42), (44) and (47) it follows that $\zeta$ lies in the sector $-5 \pi / 4 \leq \arg \zeta \leq 0$ of the $\tau$-plane at a distance from the origin not less than $|\tau|=2 r_{0}^{3 / 2} / 3$. The path $L_{\zeta}$ may be chosen as the ray

Then

$$
\tau=\zeta+\rho e^{i_{\pi} / 8} .
$$

$$
\begin{aligned}
& \left|\int_{L_{\zeta}} \tau^{-3 / 2} e^{i \tau}\left(\frac{1}{2}+i R(\tau) \tau\right) d \tau\right| \\
& \quad \leq\left|\zeta^{-3 / 2} e^{i \zeta}\right| \int_{L_{\zeta}}\left|(\zeta / \tau)^{3 / 2} e^{i(\tau-\zeta)}\right|\left(\frac{1}{2}+|R(\tau) \tau|\right)|d \tau| \\
& \quad \leq\left|\zeta^{-1 / 2} e^{i \zeta}\right| \cdot \frac{3}{2} r_{0}^{-3 / 2}\left(\sin \frac{\pi}{8}\right)^{-5 / 2}\left(\frac{1}{2}+\left|R\left(\tau^{*}\right) \tau^{*}\right|\right),
\end{aligned}
$$

where

$$
\tau^{*}=\frac{2}{3} r_{0}^{3 / 2} \sin \frac{\pi}{8}
$$

For $r_{0}=9$, the expression (51) turns out to be less than (0.89) $\left|\zeta^{-1 / 2} e^{i \zeta}\right|$.

Reinsertion into (50) shows that the quantity $O\left(s^{-1}\right)$ in (35) is indeed numerically less than one-even less than 0.89 - for $\pi / 6 \leq \arg s \leq \pi,|s| \geq 9$. There are therefore no zeros of $\psi(\bar{s})$ in this region. For $|s| \leq r_{0}$ the following extension of Rouché's theorem is needed.

Lemma 2. Let $\psi(z)$ be regular analytic in the simply connected open region $G$. Let $f(x)$ be defined and piecewise continuous on the rectifiable Jordan curve $C$ in $G$. Assume that on $C$

$$
|\psi(z)-f(z)|<|f(z)| .
$$

Denote by $\Delta$ arg $f$ the total change of arg $f(z)$ as $C$ is once described in positive direction. Then the number of zeros of $\psi(z)$ inside $C$ is the integer nearest $\Delta \arg f / 2 \pi$. Here arg $f(z)$ can - and must-be defined so that it changes continuously at points of continuity of $f(z)$ and that it jumps by less than $\pi$ at the discontinuities of $f(z)$.

Proof: The inequality (52) excludes the possibility of $\psi(z)$ having a zero on $C$. Let $C$ be defined by the equation $z=h(t), 0 \leq t<1$. Define $\alpha(t)$ as a continuous function such that

$$
\alpha(t)=\arg \psi(h(t)), \quad 0 \leq t<1 .
$$

Without loss of generality $f(h(t))$ can be supposed continuous at $t=0$. Because of (52),

$$
\operatorname{Re}\left(1+\frac{\psi-f}{f}\right)>0 \text {. }
$$


Hence,

$|\alpha(t)-\arg f(h(t))|=\left|\arg \left(1+\frac{\psi-f}{f}\right)\right|<\frac{\pi}{2}, \quad(0 \leq t<1)$

for a certain uniquely defined determination of $\arg f(h(t))$. Let this determination be called $a(t)$. The last inequality implies that $a(t)$ is continuous, except for possible jumps of less than $\pi$ at the discontinuities of $f(h(t))$, and that

$$
|[\alpha(t)-\alpha(0)]-[a(t)-a(0)]|<\pi .
$$

Since the exact number of roots of $\psi$ inside $C$ is $[\alpha(t)-\alpha(0)] / 2 \pi$, the lemma is proved.

This lemma will be applied to a contour $\Gamma$ in the $s$-plane consisting of three ares $\Gamma_{1}, \Gamma_{2}, \Gamma_{3}$ as follows: $\Gamma_{1}$ is the segment from $s=0$ to $|s|=9$ on the ray arg $s=\pi / 6 ; \Gamma_{2}$ is the circular arc defined by $s=9 e^{i \theta}$, $\pi / 6 \leq \theta \leq \pi$, and $\Gamma_{3}$ is the segment on the real $s$-axis between $s=-9$ and $s=0$. On $\Gamma_{1}$, the function $f(s)$ is to be an appropriate partial sum of the ascending power series for $\psi(s)$. On $\Gamma_{2}$, the leading term of the asymptotic series for $\psi(s)$ can be taken as $f(s)$. On $\Gamma_{3}$, the function $f(s)$ is identical with $\psi(s)$.

The ascending series for $\psi(s)$ follows from (40) by termwise integration of the series for $A i(-t)$, see [15] p. B17). Separation of the real and imaginary parts for arg $s=\pi / 6$ produces then two series with real terms of alternating sign and decreasing moduli, at least from a certain term onward. This fact makes it easy to find the number of terms needed on this ray for $f(s)$ to satisfy the inequality (52).

The necessary calculations were first carried out at the National Physical Laboratory, Teddington (England) under the direction of F. W. Olver. In this calculation $r_{0}$ was taken equal to 10 , and 13 terms of the series were needed to obtain arg $f(s)$ to two decimals of a radian on $\Gamma_{1}$.

Equivalent computations were then performed, by a somewhat different method, at the Institute for Numerical Analysis of the National Bureau of Standards, under the direction of G. Blanch. For $|s| \leq 6$ the values of $f(s)$ on $\Gamma_{1}$ were obtained by numerical integration from tables of the Harvard Computation Laboratory [18]. For $6 \leq|s| \leq 9$ the procedure was the same as Olver's. These computashow that the contribution $\Delta_{1}$ arg $f$ of $\Gamma_{1}$ to the total change $\Delta$ arg $f$ of the argument of $f(s)$ is $-3.42 \pi$. On his longer ray Olver obtained the value of $-4.11 \pi$ radians.

On $\Gamma_{2}$ the inequality (52) is satisfied by virtue of the appraisal of the remainder used for $|s| \geq 9$. The contribution $\Delta_{2} \arg f$ of $\Gamma_{2}$ to $\Delta \arg f$ is

$$
\Delta_{2} \arg f=-\frac{5}{8} \pi+\frac{\sqrt{2}}{3} 9^{3 / 2}=3.43 \pi \text {. }
$$

The corresponding value for $|s|=10$ is $4.12 \pi$.

Finally, the contribution $\Delta_{3} \arg f$ is zero, because $\psi(s)$ is real on the real axis. The condition (52) is here satisfied, since $\psi(s)$ was proved to have no zeros on the real axis.
Summarizing, it is seen that $\Delta f=0.01 \pi$ on the contour formed with the arc $|s|=9$ as well as on the contour with arc $|s|=10$ used by Olver. Reference to lemma 2 then completes the proof that $\psi(s)$ has no zeros in the sector $\pi / 6 \leq \arg s \leq 11 / 6 \pi$.

The author expresses his thanks for the advice and help he received from Dr. Olver and from Dr. Blanch in this section of the paper.

\section{The Differential Equation$$
u^{(4)}+\lambda^{2} z\left(u^{\prime \prime}+u\right)=0
$$

The occurrence of an inner friction layer is frequently regarded as a mathematical consequence of the fact that the inviscid equation is singular at the point where $w(y)-c=0$. In the case of plane Couette flow the inviscid equation, $\phi^{\prime \prime}-\alpha^{2} \phi=0$ is regular at that point, and it seems plausible that this fact is responsible for the absence of an inner friction layer implied by the unrestricted validity of formulas (10) and (11). It will, however, be shown in this section that this view is not quite correct. The total absence of an inner friction layer is actually a very special feature of the differential equation (7). This will become apparent from the discussion below, of the differential equation

$$
u^{(4)}+\lambda^{2} z\left(u^{\prime \prime}+u\right)=0 .
$$

The reduced equation $u^{\prime \prime}+u=0$ corresponding to that differential equation is also regular in the whole finite plane. But even a qualitátive a priori argument shows that some form of an inner friction layer is to be expected. The solutions of (53) are all regular at $z=0$, and their fourth derivatives must therefore vanish there. The reduced equation $u^{\prime \prime}+u=0$ has, however, only one linearly independent solution with this property. No solution $u(z, \lambda)$ of the full equation can, therefore, have a fourth derivative that converges to the fourth derivative of, say, $\cos z$ in a full neighborhood of $z=0$. But then $u(z, \lambda)$ cannot even converge uniformly on any circle $|z|=$ const, as $\lambda \rightarrow \infty$, for $u(z, \lambda)$ is an analytic function of $z$ inside the circle and it would follow that $u^{(4)}(z, \lambda)$ converges uniformly in a neighborhood of $z=0$, which is a contradiction. This remark will now be confirmed and amplified by actually solving the differential equation (53).

The usual technique of integration by Laplace contour integrals leads here to solutions of (53) of the form

$$
u(z, \lambda)=\int_{C}(i-t)^{\epsilon-1}(i+t)^{-\epsilon-1} e^{z} t+\lambda^{-2}\left(t^{3} / 3-t\right) d t,
$$

where

$$
\epsilon=1 / 2 i \lambda^{2} \text {. }
$$

Here $C$ must be a contour such that the integral exists, and that the contributions from the boundary occurring in the calculations leading to (54) vanish.

The discussion of these paths in this example is made complicated by the fact that the integrand has 


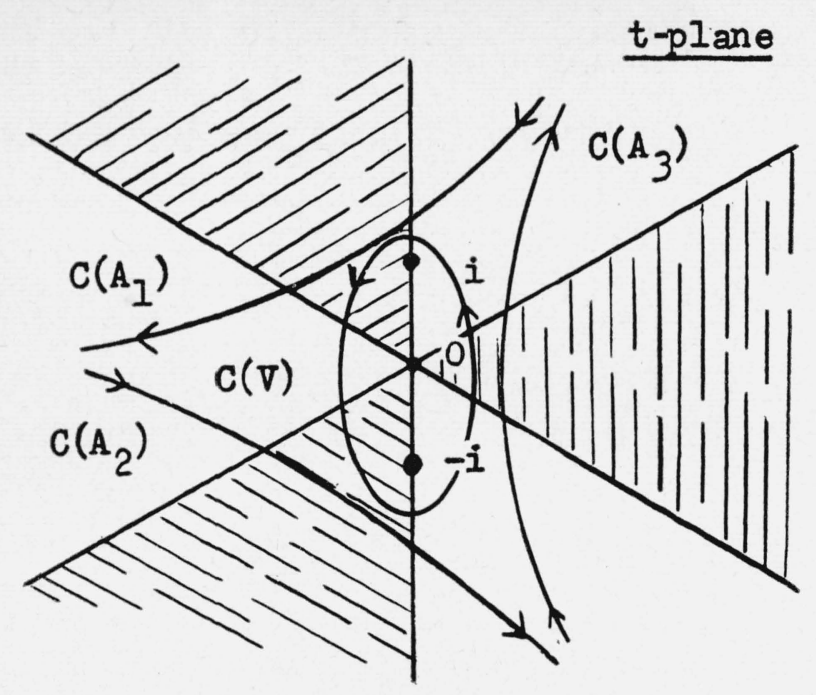

Figure 3.

branch points at $t= \pm i$. In order to bring out the analogy with the investigations in [9] seven paths are introduced, which are illustrated in the figures 3 and 4 under the assumption that $\lambda>0$.

As to the determination of the multivalued integrand to be taken the following stipulations are made: (1) Two points of the Riemann surface of the integrand are considered to be on the same sheet, if they can be joined by an arc on the Riemann surface that does not cross the segment connecting the points $t=-i$ and $t=i$. (2) $C(V) ; C\left(A_{k}\right),(k=1,2,3)$, the lower edge of $C\left(U_{3}\right)$, the upper edge of $C\left(U_{2}\right)$, and the right edge of $C\left(U_{1}\right)$ lie in the same sheet.

As before, the functions defined by (54) will be called $A_{k}, U_{k}, V$ according as $C$ is taken to be $C\left(A_{k}\right)$, $C\left(U_{k}\right)$ or $C(V)$, respectively.

From these definitions the following identities can be derived:

$$
\begin{gathered}
A_{1}+A_{2}+A_{3}=V, \\
U_{3}-U_{2}=\left(e^{\pi \lambda^{2}}-1\right) A_{1}, \\
U_{2}-U_{1}=\left(e^{\pi \lambda^{2}}-1\right) A_{3} .
\end{gathered}
$$

They result directly from an inspection of the paths concerned and the fact that the integrand in (54) is multiplied by $e^{\pi / \lambda^{2}}$, if the point $t=i$ is circled once in positive direction. The further identity

$$
U_{3}-U_{1}=\left(e^{\pi / \lambda^{2}}-1\right)\left(V-A_{2}\right)
$$

follows by a linear combination of the preceding ones.

The asymptotic calculation of the $\mathbf{A}_{k}(z, \lambda)$ for large $\lambda$ differs only in trivial details from these given in [9], and the results are exactly the same.

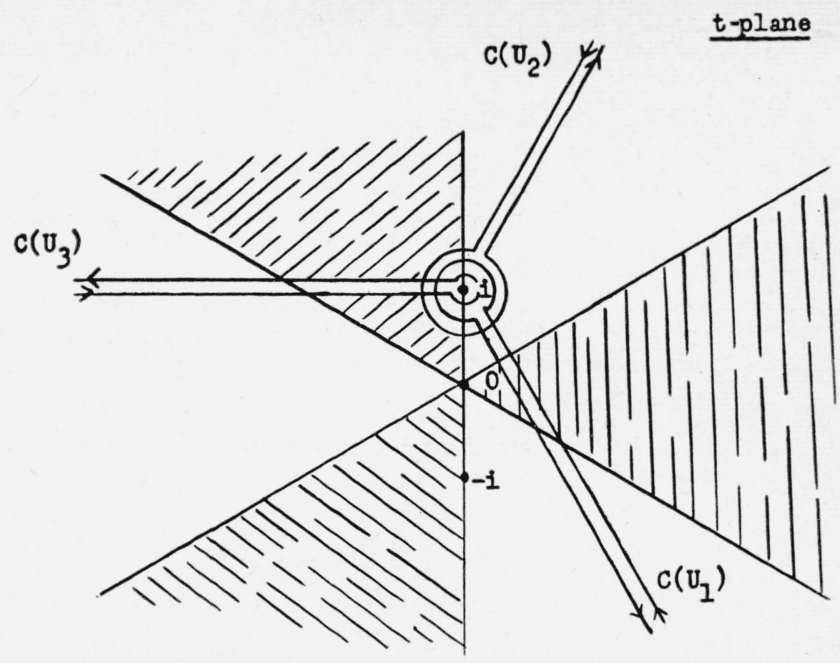

Figure 4.

For $V(x, \lambda)$ one finds without difficulty the asymptotic expression

$$
\begin{aligned}
V(z, \lambda) & =\int_{C(V)}\left(1+t^{2}\right)^{-1} e^{t z} d t+O\left(\lambda^{-2}\right) \\
& =2 \pi i \sin z+O\left(\lambda^{-2}\right)
\end{aligned}
$$

valid in any bounded domain.

For the asymptotic calculation of $U_{k}$ for $z$ in $S-S_{k}$ and $|\xi| \geq \xi_{0}>0$, one can proceed essentially as in [10], p. 12 . Since

$$
\int_{C\left(U_{k}\right)} \frac{t^{m}}{1+t^{2}} e^{t z} d t=\frac{d^{m}}{d z^{m}}\left(\pi e^{i z}\right),
$$

one has

$$
\begin{aligned}
& \frac{d^{m}}{d z^{m}}\left\{U_{k}-\pi e^{i z}\right\} \\
& =\int_{C\left(U_{k}\right)}\left[(t-i)^{\epsilon-1}(t+i)^{-\epsilon^{-1}} e^{\lambda^{-2}\left(t^{3} / 3-t\right)}-\left(1+t^{2}\right)^{-1}\right] t^{m} e^{t z} d t \\
& =\int_{C\left(U_{k}\right)}\left[e^{\lambda^{-2}\left(t^{3} / 3-t+\arctan t\right)}-1\right] \frac{t^{m}}{1+t^{2}} e^{t z} d t .
\end{aligned}
$$

The expression in brackets in the last integral is numerically less than a constant multiple of $\left|\lambda^{-2}\left(t^{3} / 3-t+\arctan _{1} t\right)\right| \max _{t \in C\left(U_{k}\right)} e^{\operatorname{Re}\left[\lambda^{-2}\left(t 3 / 3-t+\arctan _{-t}^{-t}\right]\right.}$, hence the integral is of the form

$$
\lambda^{-2} \int_{C\left(U_{n}\right)} t^{m+1} e^{t z} f(t, \lambda) d t,
$$

where $f(t, \lambda)$ is uniformly bounded on $C\left(U_{k}\right)$. Split $C\left(U_{k}\right)$ into a circle $C_{1}$ of radius $r$ about $t=i$ and two rays $C_{2}$ and $C_{3}$ going to, and coming from, infinity 
at the same angle, in two different sheets of the Riemann surface. The contribution of $C_{1}$ to the integral is $O\left(\lambda^{-2}\right)$. The contributions of $C_{2}$ and $C_{3}$ differ only by the factor $-e^{\pi / \lambda^{2}}$. Hence their sum is

$$
\left(e^{\pi / \lambda^{2}}-1\right) \int_{C_{2}} t^{m+1} e^{t z} f(t, z) d t
$$

By means of the transformation $\tau=t z$, the value of this integral is found, similarly as in [10], p. 13, to be $O\left(\lambda^{-4} z^{-m-2}\right)=O\left(\lambda^{2(m+2) / 3-4} \xi^{-2(m+2) / 3}\right)$. Hence,

$U_{k}^{(m)}(z, \lambda)=\frac{d^{m}}{d x^{m}}\left(\pi e^{i z}\right)+O\left(\lambda^{-2}\right)+O\left(\lambda^{2(m+2) / 3-4} \xi^{-2(m+2) / 3}\right)$,

for $z$ in $S-S_{k}$ and $|\xi| \geq \xi_{0}>0, m \geq 0$. The remainder in (62) is $O\left(\lambda^{-2}\right)$ for all $m$, if $z$ is bounded away from zero. Otherwise, the remainder tends to zero for $m \leq 4$, but not necessarily for $m>4$.

In order to deal with the higher derivatives and also with the case $|\xi| \leq \xi_{0}$, observe that the contribution to (54) from the path $C_{1}$ is in any case equal to

$$
\frac{d^{m}}{d x^{m}}\left(\pi e^{i z}\right)+O\left(\lambda^{-2}\right)
$$

The contribution of the two rays $C_{2}$ and $C_{3}$ is, if $\sigma$ is defined by $\lambda^{-2} t^{3}=\sigma^{3}$,

$$
\begin{aligned}
& \left(e^{\pi / \lambda^{2}}-1\right) \lambda^{2(m-1) / 3} \int_{C_{2}^{*}} \exp \left\{\sigma^{3} / 3-\right. \\
& \left.\lambda^{-4 / 3} \sigma+\lambda^{-2} \arctan \sigma \lambda^{2 / 3}+\eta \sigma\right\} \frac{\sigma^{m}}{\lambda^{-4 / 3}+\sigma^{2}} d \sigma,
\end{aligned}
$$

and a short argument, omitted here, shows that this is equal to

$$
\lambda^{2(m-1) / 3-2} \int_{C_{2}^{*}} e^{\sigma^{3} / 3+\eta \sigma} \sigma^{m-2} d \sigma\left[1+O\left(\lambda^{-2 / 3}\right)\right],
$$

where $C_{2}^{*}$ is a ray in the $\sigma$-plane along which $\operatorname{Re} \sigma^{3}<0$ and whose initial point has at least the distance $\left|\lambda^{-2 / 5}\right|$ from the origin.

In $S_{k}$, finally, the asymptotic behavior of $U_{k}(z, \lambda)$ follows immediately from the identities (57) to (59), the asymptotic properties of the $A_{k}(z, \lambda)$, and $(62)$. It is then seen that $\mathrm{U}_{k}(z, \lambda)$ diverges for $\xi \mid \geq \xi_{0}$ in the whole interior of $S_{k}$ in the same fashion as the corresponding functions in [9] and [10], except for a factor $e^{\pi / \lambda^{2}}-1=O\left(\lambda^{-2}\right)$.
Thus, there is in this problem an "inner friction layer" of finite thickness for $|\xi| \geq \xi_{0}$, and also a friction layer at $z=0$. The latter occurs only in the derivatives of order four or higher.

\section{References}

[1] L. Prandtl, The mechanies of viscous fluids, Aerodynamic theory, III, Reprint edition (Calif. Inst. of Tech., Pasadena, Calif., 1943).

[2] J. L. Synge, Hydrodynamic stability, Semicentennial publications of the Am. Math. Soc. 2, 227-269 (1938).

[3] C. C. Lin, On the stability of two-dimensional parallel flows, Quart. Appl. Math. 3, 117-142, 218-234, 277301 (1945).

[4] W. Tollmien, Laminare Grenzschichten, Hydro- and aerodynamics, Fiat review of German science 19391946 (Wiesbaden, 1948)

[5] G. B. Schubauer and H. K. Skramstadt, Laminar boundary-layer oscillations and stability of laminar flow, J. Aeronaut. Sci. 14, 69 (1947).

[6] H. L. Dryden, Recent advances in the mechanics of boundary-layer flow, Advances in applied mechanies, I (New York, N. Y., 1948).

[7] W. Tollmien, Asymptotische Integration der Stoerungsdifferentialgleichung ebener laminarer Strönungen bei hohen Reynoldschen Zahlen, Z. Angew. Math. Mech. 25, 33-50; 27, 70-83 (1947).

[8] W. Wasow, The complex asymptotic theory of a fourth order differential equation of hydrodynamics, Ann. Math. 49, 852-871 (1948).

[9] W. Wasow, A study of the solution of the differential equation $y^{(4)}+\lambda^{2}\left(x y^{\prime \prime}+y\right)=0$ for large values of $\lambda$, Ann. Math. 52, 350-361 (1950).

[10] W. Wasow, Asymptotic solution of the differential equation of hydrodynamic stability in a domain containing a transition point, Ann. Math. (to be published).

[11] Lord Rayleigh, Further remarks on the stability of viscous fluid motion, Sci. Pap. VI, 341-349 (Cambridge, 1920).

[12] C. L. Pekeris, On the stability problem in hydrodynamics, Proc. Cambridge Phil. Soc. 32, 55-66 (1936).

[13] C. S. Morawetz, The eigenvalues of some stability problems involving viscosity, J. Rat. Mech. Analysis 1. No. 4, 579-603 (1952).

[14] H. Jeffreys and B. S. Jeffreys, Methods of mathematical physies (Cambridge, 1946).

[15] J. C. P. Miller, The Airy Integral, Mathematical tables, Part B, British Ass. for the Advancement of Science (Cambridge 1946).

[16] H. Weber, Zur Theorie der Besselschen Funktionen. Math. Ann. 37, 404-416 (1890).

[17] G. N. Watson, Theory of Bessel functions, $2 \mathrm{~d}$ ed. (Cambridge, 1948).

[18] Tables of the modified Hankel functions of order onethird and of their derivatives (Computation Laboratory, Harvard University, Cambridge, Mass., 1945).

Los Angeles, November 20, 1952. 